Revue

de Sémantique

et Pragmatique
Revue de Sémantique et Pragmatique

37 | 2015

Sémantique et pragmatique

\title{
Agent passif en PAR versus sujet actif : les dessous d'un « contraste »
}

\section{Badreddine Hamma}

\section{(2) OpenEdition}

\section{Journals}

Édition électronique

URL : http://journals.openedition.org/rsp/1177

DOI : $10.4000 /$ rsp. 1177

ISSN : 2610-4377

Éditeur

Presses universitaires d'Orléans

\section{Édition imprimée}

Date de publication : 1 juin 2015

Pagination : 61-83

ISSN : 1285-4093

\section{Référence électronique}

Badreddine Hamma, «Agent passif en PAR versus sujet actif : les dessous d'un « contraste » ", Revue de Sémantique et Pragmatique [En ligne], 37 | 2015, mis en ligne le 01 juin 2016, consulté le 27 juin 2020. URL : http://journals.openedition.org/rsp/1177 ; DOI : https://doi.org/10.4000/rsp. 1177 


\section{AgENT PASSIF EN PAR VERSUS SUJET ACTIF: LES DESSOUS D'UN «CONTRASTE »}

Badreddine Hamma

Université d'Orléans \& UMR 7270, (LLL) Laboratoire Ligérien de Linguistique

\section{INTRODUCTION}

L'objet de cette étude est de questionner les enjeux discursifs sous-jacents à l'emploi du «complément d'agent » en par dans une phrase passive, par opposition au «sujet » correspondant dans une phrase active, en vue de vérifier si ces deux constituants phrastiques, qui, certes, entretiennent des rapports légitimes sur les plans sémantique et syntaxique, conservent ou non une certaine continuité sur le plan pragmatique, au-delà des effets de topicalisation résultant du passage d'une phrase active $(P A)$ à une phrase passive $(P P)$, connus dans la littérature sur le passif sous les noms de «promotion de l'objet au rang de sujet » (cf. Gaatone, $1998: 31)$ ou de «marquage iconique d'un changement de saillance » (Kleiber, 1993 : 119). Un exemple, comme (1a), rapporterait de ce point de vue, « ce que l'on fait à la voiture » (par Luc est d'ailleurs perçu comme « facultatif »), là où dans la version active (1), c'est plutôt « ce que fait Luc à la voiture » qui serait mis en avant (contrairement à (1a), Luc y est un constituant « essentiel ») :

1. Luc a amené la voiture au garage.

1a. La voiture a été amenée par Luc au garage.

Mais dans cette tentative de cerner les différences qui opposent ces deux constituants, nous ne nous arrêterons pas aux effets de topicalisation produits, qui sont d'ailleurs largement décrits, nous allons sonder de nouveaux aspects 
discursifs qui présentent, à notre sens, un intérêt capital dans ce type de construction. Ce qui motive cette étude est le fait que le rapprochement entre « agent passif » $(A P)$ et « sujet actif » $(S A)$ est parfois poussé à l'extrême dans la tradition, au point de les considérer comme des « synonymes ». Nous verrons qu'une telle assimilation est trompeuse et ne résiste pas face à l'examen attentif des interactions observables dans les énoncés considérés. En effet, les correspondances établies entre $A P$ et $S P$ semblent faire abstraction d'une valeur importante et dont on ne peut faire l'économie selon nous, à savoir l'expression d'un certain « contraste » dont se revêt l'AP dans une $P P$ ( $c f$. infra), et qui disparaît dans la version active avec le $S A$ correspondant, bien entendu à lexique constant et sans l'intervention d'autres faits linguistiques à valeur contrastive, comme le clivage ou le pseudo-clivage, le détachement à gauche et les appositions.

Notre propos concernera ainsi les $P P$ à l'oral dites «longues » ou « achevées » (Dubois, $1967: 24-25$ ), c'est-à-dire celles qui se construisent avec un « complément d'agent ». Ce complément d'agent est généralement un syntagme nominal $\left(N_{l}\right)$ introduit par la préposition $\mathrm{par}^{l}$, dans la relation $N_{O} V$ par $N_{l}$ et que l'on a coutume de relier à sa contrepartie active $\left(N_{O} V N_{l}\right)$, tenue pour phrase de base de la $P P$. L'intérêt porté ici aux données orales dans leur contexte est motivé par trois raisons bien précises : la première est que le présent travail entend compléter un travail déjà établi sur l'écrit (2005, 2006 et 2007) ; la deuxième raison a trait au fait qu'une approche dialogique, transphrastique et macrosyntaxique ( $c f$. Blanche-Benveniste, 1990 ; Berrendonner, 1990), dépassant le cadre de la phrase monologale, simplifiée et mobilisant, ipso facto, les différents phénomènes d'interaction qu'offre le discours oral : dialogisme, marques polyphoniques, attitudes énonciatives, prosodie, etc., permet de mieux cerner les usages de ces énoncés. Quant à la troisième raison, elle concerne la rareté, voire la quasi-absence de travaux sur le passif en français parlé, exception faite des deux études de Blanche-Benveniste (1988 et 2000).

Les bases de données orales utilisées dans ce travail ont vocation, d'ailleurs,

1 Nous admettons avec les linguistes ayant travaillé sur le passif que les compléments d'agent introduits par la préposition de revoient davantage à une propriété de N1 ou à une modification du verbe qu'à un «vrai » complément d'agent en par dans un contexte processif ; on parle alors de «passif adjectival » ou de « variété de génitif » (Helland, 2002 ; Martin, 2002) : Marie est aimée de tous/de tout le monde; aimé de N1 ici s'interprète comme une propriété (pouvant commuter avec un adjectif) = «Marie est populaire/appréciée » et cela n'évoque pas a priori l'idée de «contraste » (vs La voiture est amenée au garage par $L u c$ ). Par ailleurs, les emplois de de ont des affinités avec le sens « générique » et sont plus naturels au pluriel ou aussi avec un article zéro : ??Marie est aimée de Max; Max est passionné del ??du foot (voir Martin, op. cit., pour une étude détaillée comparant l'emploi de ces deux prépositions). 
à susciter et à favoriser d'une manière indirecte ces interactions à travers les choix théoriques et les techniques d'enquêtes qui les sous-tendent ; ce sont, ici, les ESLO («Enquêtes Socio-Linguistique à Orléans » du Laboratoire Ligérien de Linguistique - LLL ; cf. Eshkol et al., 2011, pour plus de détails sur les corpus orléanais) et les CLAPI ( Corpus de LAngue Parlée et Interaction » de l'équipe «Interaction, Corpus, Apprentissage et Représentations - ICAR; cf. Balthazar \& Bert, 2005, pour plus de détails sur les corpus lyonnais).

\section{UNE IDENTITÉ SÉMANTIQUE PASSIVE-ACTIVE MISE EN CAUSE}

L' « agent passif » et le « sujet actif » sont traditionnellement considérées comme des formes équivalentes et consubstantielles : outre les différentes grammaires de référence où les $P A$ et les $P P$ sont appréhendées ensemble comme deux éléments complémentaires, qui (r)appellent l'un l'autre du point de vue formel et sémantique et qui présument que les dernières sont dérivées des premières, on peut citer aussi certains travaux sur le passif relevant ou s'inspirant de la grammaire générative transformationnelle et qui stipulent, en gros, que ces deux constructions sont issues d'une même "phrase de base »; la question du sens, quant à elle, se réduit souvent aux « rôles thématiques » assignés aux entités en jeu, et les usages effectifs de ces énoncés sont quasi absents, puisque ce sont souvent des phrases forgées, réduites à ce qui est jugé essentiel dans ce type de constructions. Les différences relevées entre une $P A$ et une $P P$ sont vues en général comme purement «stylistiques » ou « formelles », n'affectant pas le fond de la phrase : on n'aurait qu'une simple permutation des arguments et un changement morphologique concernant la « voix » verbale ( $c f$. Chomsky, 1957 trad. 1969 : 83-88 ; Ruwet, 1967 : 190-192 ; Strunck Sachs, 1967 : 441). Ces principes de la grammaire générative ont été repris par la suite par la linguistique cognitive, qui a entériné globalement les thèses de la « synonymie », d'un côté, entre $P A$ et $P P$, de l'autre, entre $S A$ et $A P$, tout en reconnaissant certaines disparités mineures. Ainsi, Jackendoff (1997), entre autres linguistes, considère, conformément à ce qui est préconisé dans le programme minimaliste chomskyen, qu'à une même "structure conceptuelle » $(C-S)$, du type (2), convertie selon des règles syntaxiques et lexicales en « structures profondes » $(D-S)$ en (3), peuvent correspondre différentes «phrases de surface » $(S-S)$, dérivées selon des règles transformationnelles spécifiques ( $c f$. flexion verbale et topicalisation, ici), ce qui donne (4) ou (5) : soit c'est l'« agent » qui constitue le thème de la phrase, soit c'est le «patient », et les changements conséquents à chaque fois sont jugés formels :

2. (C-S) EVENT [AGENT, PATIENT/UNDERGOER]

3. ${ }_{(D-S)} P\left[\left[_{S N}\right.\right.$ Chat], [SV $\left[_{S N}\right.$ souris], [V manger $\left.\left.\left._{(T E N S E)}\right]\right]\right]$

4. (S-S) $P_{1}\left[S N\right.$ (SUBJECT) La chat], ${ }_{S V}$ [V mange [SN (OBJECT) la souris]]

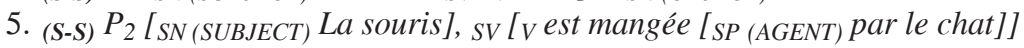


Dans la même optique théorique, certaines expériences psycho-cognitives sont invoquées afin d'appuyer les thèses de la synonymie entre $P A-P P$ ( $c f$. Strunck Sachs op.cit. : 438-439) : il est demandé à des sujets anglophones de restituer de mémoire des informations entendues sous forme de phrases à la forme active, comme en (6), en leur soumettant après l'écoulement d'un certain temps de nouvelles phrases proches des premières du point de vue du sens et de la forme, dont des phrases au passif, comme en (6a):

6. He sent a letter about it to Galtleo, the great Italian scientist.

6a. A letter about it was sent to Galfleo, the great Italian scientist (Strunck Sachs, ibid.).

Il ressort de ces expériences que les sujets interrogés ont tendance à confondre les phrases du type (6) et les phrases du type (6a); ce qui prouverait ainsi, selon l'auteur, que l'information retenue aurait pu être dite, dans l'une ou l'autre des deux formes, indifféremment, sans perte de sens. Or, une telle allégation paraît fortement contestable comme nous allons le voir dans ce qui suit, dans la mesure où il n'est pas tenu compte de l'usage effectif des formes comparées et que la comparaison n'y concerne que les rôles sémantiques des actants.

Le traitement des $P A$ et des $P P$ dans le discours didactique est, d'ailleurs, le meilleur témoin des thèses « synonymistes » $(c f$. Leduc-Adine, 1977 ; Le Bellec \& Hamma,2011); en effet, les exercices et leçons liés aux relations actif-passif reposent habituellement sur ce principe d'identité sémantique et consistent, en général, soit à conjuguer des verbes à la « voix » active ou passive (LeducAdine, op.cit. : 90), soit à passer de l'une à l'autre - machinalement - en ne posant comme condition que le fait d'avoir un verbe transitif avec un certain sens « concret». En conséquence, l'acceptabilité des phrases obtenues dans ces manuels scolaires laisse à désirer du point de vue de l'usage ( $c f$. la critique faite par Le Bellec \& Hamma, op.cit. à propos d'exemples du type : Alain regarde avec intérêt toutes les jolies femmes qui passent $\rightarrow$ ?Toutes les jolies femmes qui passent sont regardées avec intérêt par Alain ${ }^{02}$ ).

Par ailleurs, quand la « synonymie » actif-passif est contestée dans la littérature consacrée au passif, les raisons invoquées par les linguistes qui s'y sont intéressés de près concernent davantage les changements opérés au niveau du sens et de la construction des unités lexicales, prises isolément, et leur impact global sur la phrase, mais rarement au niveau de la praxis ; cela peut concerner les contraintes pesant sur les distributions lexicales attestées : il est difficile d'avoir, par exemple, des constructions ne respectant pas les règles de souscatégorisation lexicale ( $c f$. Jackendoff, 2008 : 95-96) ; on aura ainsi en anglais

\footnotetext{
2 Mimran, et al. (2002), Grammaire expliquée du français, CLE International.
} 
The bed was slept in/on mais jamais *The bed was slept under/beside : le procès sleep under/beside n'étant pas attesté à la forme passive. Il y a également le problème posé par certains quantifieurs ( $c f$. Muller, 1977 : 60-63) : Toutes les filles aiment un garçon $\boldsymbol{v s}$ Un garçon est aimé par toutes les filles ou aussi La cible n'a pas été atteinte par beaucoup de flèches vs Beaucoup de flèches $n$ 'ont pas atteint la cible; ou celui des blocages transformationnels causés par l'utilisation d'une forme verbale « abstraite»: Marie est dévorée par la jalousie $\rightarrow$ *Marie est dévorée; Les matchs de foot rasent/barbent Luc $\rightarrow *$ Luc est barbé/rasé ( $c f$. Mathieu, 1993 : 35 ; voir également Leclère, 1993 : 10), sans oublier les changements au niveau des valeurs aspectuelles impliqués par les formes passives : il est souvent constaté que le procès de la phrase passe de l'« inaccompli » à l' « accompli » (Carlier, $2002: 41)$, ou encore les effets de topicalisation entraînés par la permutation des actants (Gaatone, 1998 : 17). De fait, on a tendance à faire état des changements produits tout en reconnaissant, en revanche, une certaine « synonymie » entre $P A$ et $P P$; c'est ce qui amène Helland à parler de « (quasi-) synonymie » (2002 : 21). De même, Gaatone $(1998, i b i d$.) considère qu'il y a malgré tout une certaine synonymie entre actif et passif, abstraction faite de l'effet du changement de points de vue, résultant de la permutation des actants et que ces changements sont, d'après lui, de la même nature que les nuances observables, par exemple, entre une « question avec ou sans inversion du sujet » ou entre une «clivée et une non clivée » (ibid.). Notons cependant que la contestation d'une éventuelle «synonymie » actif-passif, dans ces travaux, ne concerne guère les changements observés au niveau de leurs usages et des intentions discursives en jeu, en tous cas, n'a pas de liens avec l'idée de « contraste » dont il est question dans ce travail. Or, selon nous, c'est l'un des points constants qui font toute la différence dans les rapports actifpassif mobilisant un complément d'agent en par : nous pensons que ces deux formes sont loin d'être équivalentes (au-delà des effets décrits ci-dessus) et que l'utilisation de l'une d'entre elles est, en principe, de nature à exclure l'autre. En effet, l'AP et non le $S A$ donne à voir, du point de vue pragmatique, une attitude particulière chez le locuteur qui se traduit à travers l'expression d'un certain « contraste » que l'on doit essentiellement à l'usage de la préposition par (Hamma, 2005, 2006, 2007). Ainsi, l'« agent » désigné Y (dans par $N_{l}$ ) est opposé implicitement ou explicitement à d'autres agents possibles, $\mathbf{X}$, paraissant plus vraisemblables que $\mathbf{Y}$. De fait, le recours à la forme passive achevée est assimilable à une construction négative (l'énoncé stipule « l'agent n'est pas $\mathbf{X}$, mais $\mathbf{Y} »)$. La question ne se pose pas dans les énoncés passifs «tronqués » : l'agent étant tu (par trop évident ou parce qu'il est inconnu ou que l'on cherche à le laisser dans l'ombre), n'implique pas de contraste ; c'est plutôt le procès en tant que « résultat» qui est mis en avant. En d'autres termes, les énoncés passifs achevés informent du positionnement de l'énonciateur (E) vis-à-vis de ce qu'il 
énonce : du point de vue de $\mathbf{E}$, l'agent $\mathbf{X}$ peut paraître comme plus «évident » et plus « crédible » que celui qu'il consigne comme le « vrai agent» (Y) dans son énoncé. Les attentes ou les prévisions du co-énonciateur $(\mathbf{C}-\mathbf{E})$ se trouvent ainsi démenties et invalidées par la «singularité » de la situation rapportée par E, lui-même. Ce dernier apporte une précision concernant l'agent qu'il juge nécessaire, en devançant un raisonnement fondé globalement sur une certaine « logique commune » de type « prédictif », une sorte de «bon sens collectif», un «ON-locuteur» (Anscombre 2005 et François 2005). Le locuteur écarte une possibilité trop évidente $(\mathbf{X})$ et rétablit les faits, en apportant des précisions ou des rectifications qu'il juge nécessaires concernant un agent $\mathbf{Y}$. L'énoncé au passif avec un agent explicite permet ainsi, selon $\mathbf{E}$, de «mettre les points sur les i », de lever une ambigüité éventuelle, pressentie ou constatée chez $\mathbf{C}$-E.

Cette valeur « contrastive » associant $\mathbf{Y}$ à $\mathbf{X}$ est, en fait, particulièrement patente dans les conversations orales et, de ce point de vue, elle peut très bien ne pas être décelable dans des phrases forgées monologales ou relevant d'un écrit décontextualisé, semblable à celui que l'on rencontre, par exemple, dans certains manuels scolaires ( $c f$. Le Bellec et Hamma, op.cit.) ou même dans certaines grammaires de référence et où les interactions conversationnelles sont neutralisées, vu que l'on s'y intéresse avant tout à la manière dont s'opèrent les transformations active-passive ; or, il va sans dire, que l'intérêt de ce tour discursif ne se réduit pas à être un simple exercice scolaire ou à une manipulation linguistique. Ce fait de langue, si particulier, met en scène des interactions énonciatives très singulières, dont nous nous proposons de rendre compte dans le présent travail.

\section{AGENT PASSIF VERSUS SUJET ACTIF ET NOTION DE « CONTRASTE »}

L'« agent » dans la phrase passive est perçu dans la tradition comme le « sujet sémantique réel », qui, suite à une opération de passivation, cède sa place initiale de thème, à gauche du verbe, au « patient » $\left(N_{l}\right)$ de la phrase active dans la relation $N_{0} V N_{l}$. Certains postulent, d'ailleurs, que la forme active est « primaire » et que l'acquisition de la forme passive chez les enfants vient après ( $c f$. Ud Deen, 2011 : 155). Corollairement, le $N_{l}$ la voiture dans une phrase comme Luc a amené la voiture au garage devient le "sujet grammatical » $\left(N_{0}\right)$ dans La voiture a été amenée par Luc au garage, sans devenir le «sujet sémantique » et l'agent passe alors à droite du verbe et devient, dans de nombreux cas, facultatif. Cet aspect « subalterne » de l'agent dans la PP est considéré par Desclés \& Guentechéva (1993 : 80) comme une « dégradation », une sorte d' «évincement de l'agent» sans nier son existence : «La passivation a une première fonction discursive : elle tend à évincer l'agent de la relation prédicative de base sans pour autant supprimer l'affirmation de son existence ». Or, ici, notre recherche tend à montrer l'effet inverse, sous réserve de partir des 
$P P$ avec agent et de tenter de supprimer ce dernier, puisque, comme nous l'avons annoncé plus haut, notre étude concerne avant tout les cas où un " complément d'agent » en par est réalisé dans les données recueillies, mais nous aurons à les confronter aussi à des cas où celui-ci serait effacé et, bien entendu, à la version active correspondante avec un sujet actif.

Il est communément admis que quand une information est évidente, il est inutile de la mentionner, en vertu des lois d' « informativité », de "quantité » et de « pertinence » (Grice, 1975). De facto, l'information que véhicule l'AP dans un exemple comme (7) est a priori superfétatoire si l'on se fiait aux « savoirs partagés » (« selon la doxa, il va de soi que l'on se fait soigner les dents par un dentiste ») et l'énoncé devient de ce point de vue bizarre :

7. ?Je vais me faire soigner les dents par un dentiste.

Si le procès «aller se faire soigner les dents » constitue un prédicat tout à fait naturel (le locuteur peut annoncer ce qu'il a l'intention de faire), la précision concernant le « complément d'agent » par le dentiste paraît en revanche redondante, puisque, par définition, le SN un dentiste renvoie à l' agent $\mathbf{X}$ attribué par défaut pour une action du type «se faire soigner les dents » en termes de sélection sémantique, et rien ne l'impose dans l'énoncé, ni s'y oppose. Dans ce type de situations, le SP par $N_{l}$ sera tout simplement omis et la version en (7a) suffirait; on comprendrait par défaut que le locuteur va se faire soigner les dents par un dentiste. On peut dire que ce premier cas correspond à la fonction d' ' évincement de l'agent » dont parlent Desclés \& Guentechéva (op.cit.) et que semblent partager beaucoup de langues naturelles. Notons, cependant, que ce n'est pas l'apanage des PP puisque le fait de dire Je vais chez le dentiste dans ce type de situations dispense en principe de préciser (pour) me faire soigner les dents (à moins de vouloir apporter une précision sur le type de soins, comme en (7b) :

7a. Je vais me faire soigner les dents.

7b. Je vais chez le dentiste $(\emptyset+?$ ?me faire soigner les dents + faire un détartrage + me faire poser un bridge + me faire arracher la dent de sagesse...).

Ceci dit, il existe deux autres situations possibles où la phrase Je vais me faire soigner les dents peut admettre un complément d'agent sans que l'information choque ou paraisse superflue : pour ce faire, il faudrait que l'agent précisé ne soit pas unique, ni "prédictible », ou conforme aux attentes et aux savoirs partagés (il faut qu'il renvoie à un agent du type $\mathbf{Y}$ et non $\mathbf{X}$ ), dans, par exemple, (7c) :

7c. Je vais me faire soigner les dents par le gardien de l'immeuble / mon ami le garagiste / ma grand-mère.

Certes, (7c) renferme une étrangeté : l'agent $\mathbf{Y}$ n'y est pas conventionnel. Mais, c'est bien cette étrangeté qui légitime cette précision du point de vue pragmatique ; il s'agit d'une information nouvelle, supposée ignorée par 
l'interlocuteur et mérite, selon l'énonciateur, d'être mentionnée, étant donné qu'elle ne va pas de soi justement. De ce point de vue, (7c) devient plus naturel que (7). Ainsi, l'interlocuteur comprendrait sans peine que le locuteur, dans (7c), voulait lui faire remarquer le fait que l'agent désigné le gardien de l'immeuble (Y), par exemple, pour l'action " soigner les dents », quoique paraissant " inapproprié », demeure bel et bien le bon agent impliqué dans cet énoncé et que un dentiste, l'agent " prévisible » $\mathbf{X}$ est, en revanche, une inférence à exclure. On pourrait gloser cet énoncé ainsi « contrairement à ce à quoi l'on peut s'attendre, le locuteur se fait soigner les dents non par un dentiste, mais par le gardien de l'immeuble! »). Notons que la version active Le dentiste est en train de soigner les dents de Luc correspond, quant à elle, à une prédication plus naturelle : que l'on parle de ce que fait le dentiste (parmi tout ce qu'il a à faire) ou de celui qui constitue le «bénéficiaire » de l'action du dentiste ( $L u c)$, ou des deux ensemble, dans la formulation active, ces informations restent pragmatiquement pertinentes, ce qui constitue un argument supplémentaire contre l'hypothèse de la synonymie.

La deuxième situation où Je vais me faire soigner les dents peut admettre un complément d'agent, suppose un autre type de scénario : le locuteur avait l'habitude de se faire soigner les dents, non par un dentiste (agent $\mathbf{X}$ ) mais par sa grand-mère, par exemple, ou un ami qui s'y connaît ou tout autre personne n'étant pas habilitée, ni supposée connaître l'art et les techniques des soins dentaires (correspondant à l'agent $\mathbf{Y}$ ) et l'information annoncée par le complément d'agent par un dentiste, dans (7) ci-dessus, devient alors naturelle si la situation évoque une certaine « rupture » avec ce qui précède. Dans ces différents cas de figure, l'attitude du locuteur est la même : elle souligne une situation singulière mettant en scène un « contraste » entre un agent $\mathbf{X}$ «attendu/ ayant les propriétés requises pour être sélectionné sémantiquement comme meilleur agent possible » et un agent $\mathbf{Y}$ « inattendu/ invraisemblable » et, en fin de compte, il s'avère que c'est $\mathbf{X}$ qui se trouve écarté pour laisser sa place à l'agent $\mathbf{Y}$, que la doxa ne favorise pas forcément.

Notons, toutefois, que dans le cas où un énoncé comme (7) existerait, il se trouvera en principe accompagné d'éléments co(n)textuels facilitant son interprétation dans un échange naturel (d'où notre choix, ici, de travailler sur des données orales, issues d'échanges conversationnels) ; cela suscite des commentaires, des gloses, des réactions et différents types d'attitudes qui mettent en évidence les éléments concourant à l'expression de ce " contraste » entre $\mathbf{X}$ et $\mathbf{Y}$, des différents interactants : l'énonciateur cherchera à justifier ses propos et à les rendre cohérents à travers différents types d'ajouts, par exemple, des modifieurs adverbiaux se rattachant à la phrase entière comme en (7d) ou des modifieurs se rattachant au $N_{l}$, comme vrai dans (7e) : 
7d. Je vais me faire soigner les dents, pour une fois/cette fois-ci, par un dentiste!

7e. Je vais me faire soigner les dents par un vrai dentiste!

Et le co-énonciateur confirmera la connivence ou alors exprimera sa surprise en émettant par exemple des commentaires qui sont de nature à permettre de mieux comprendre l'énoncé de départ et qui serait passé pour bizarre quand il apparaît seul ; ainsi, il pourrait s'enquérir du secret de cette rupture ou évoquer explicitement l'autre «agent» $(7 \mathrm{f}-\mathbf{B})$ et $(7 \mathrm{~g}-\mathbf{B})$. Et c'est ainsi qu'une prise en compte des aspects dialogiques et macrosyntaxiques dans ces échanges entrent en jeu et deviennent indispensables.

7f. A : Je vais me faire soigner les dents par un dentiste.

B : Ta grand-mère ne veut plus te voir ?! / Tu faisais comment avant !!?

7g. A : Je vais me faire soigner les dents par un dentiste.

B : (Ah bon ?!) Qu'est-ce qui t'arrive? / Tu t'es fâché avec ton ami le garagiste?

En corollaire, quand d'autres agents potentiels $(\mathbf{X})$ sont concernés, étant d'une meilleure crédibilité, et que le « vrai » agent (Y), quant à lui, ne semble pas évident ou est susceptible d'être pris pour un autre qui serait « plus pertinent », la notion de « contraste » $\mathrm{s}$ 'invite. Ce contraste peut être assimilé à un paradigme d'agents possibles et dont l'agent désigné, dans l'énoncé considéré, soit n'en fait pas forcément partie en termes de sélections sémantiques, soit en est le membre le moins représentatif, le moins attendu, du point de vue du locuteur. Notons que le caractère « inattendu », «non-canonique » ou « peu évident » imputé à l'agent en par, dans cette étude, ne sont que des effets de sens découlant de la notion - centrale - de « contraste » que nous défendons ici. En effet, ce contraste peut très bien relever d'une simple opposition paradigmatique ayant pour effet une singularisation de l'agent Y (l'agent réalisé en surface), supposé « inconnu », en le distinguant du lot des agents possibles (X) : La lune est attirée par la terre sous l'effet de la gravitation (la terre par opposition aux autres planètes) ; Ce beau portrait a été réalisé par Luc (Luc par opposition aux autres personnes concernées) ; c'est aussi la raison pour laquelle l'agent est souvent utilisé pour désigner les inventeurs, les concepteurs, les auteurs, etc. (avec ou sans expression du procès) : L'éducation sentimentale ((a été) écrit) par Flaubert; Le vaccin contre la rage ((a été) mis au point) par Pasteur; Guernica ((a été) peint en 1937) par Pablo Picasso, etc.

La preuve que les phrases passives avec un agent en par s'inscrivent toujours dans cette optique « contrastive » est qu'elles ne sont pas tout à fait naturelles comme réponse devant une question du type Qui a réparé la voiture?, laquelle peut laisser entendre que l'interlocuteur n'a pas la moindre idée a priori de qui en est l'auteur et rend ainsi l'emploi de $\operatorname{par} N_{l}$ injustifié, ce qui corrobore encore une fois nos hypothèses :

8. ?Qui a réparé la voiture? => La voiture a été réparée par Luc. 
Il n'est pas utile, ni naturel, en effet, de reprendre l'objet support de l'interrogation ( $L a$ voiture) dans la réponse ; un tel échange n'est a priori attesté que dans certains outils scolaires, à tort, à travers certains drills visant à inculquer des règles grammaticales aux élèves d'une manière irréfléchie et machinale. La réponse la plus naturelle, et qui est aussi la plus économique (respectant la loi de « quantité »), relativement à cette question serait un énoncé, du type (8a) ou éventuellement (8b) :

8a. A : Qui a réparé la voiture? B : (C'est) Luc!

8b. A : Qui a réparé la voiture? B : C'est Luc qui l'a réparée.

Par ailleurs, pour qu'une question soit elle-même plus naturelle relativement à une réponse à la forme passive (La voiture a été réparée par $L u c$ ), il faudrait que l'agent soit saillant par une mise en focus dans la formulation de la question, par exemple, comme en (9), ou se contenter d'évoquer seulement le complément d'agent $\operatorname{par} N_{l}$ :

\section{A : Par qui la voiture a été réparée?}

B : (?La voiture a été réparée) Par Luc / C'est Luc qui l'a réparée.

Or l'une des fonctions de la mise en focus (ici, à travers l'extraction en c'est...qui) est justement le "contraste ». Notons au passage qu'une réponse du type (8a) n'est pas naturelle avec une question comme (9) ; en revanche, si (8b) l'est, c'est parce qu'il renferme justement une extraction permettant de récupérer la notion de "contraste » dans C'est Luc qui l'a réparée. Mais la situation la plus ordinaire de l'énoncé La voiture a été réparée par Luc est, sans doute, quand on contraste le $\mathrm{N}_{1}\left(l^{\prime} A P\right)$, Luc (l'élément $\mathbf{Y}$ ), avec d'autres agents possibles du même type (X), dans le cadre d'une énumération, ou d'une distribution de tâches, en (9a) :

9a. La voiture a été réparée par Luc alors que la moto c'est Marie qui s'en est occupée.

De plus, quand le sujet dont on s'enquiert est mis en focus par «par qui », comme en (9), cela sous-entend que celui qui pose la question présuppose que l'agent en question fasse partie d'un ensemble, plus ou moins fermé, d'agents possibles $(\mathbf{X})$, la question posée signifierait quelque part «Qui a fait ça parmi Luc, Marie ou Jean ?», ou toute personne présumée s'inscrire dans un groupe, dans une pluralité ; en effet, il est difficile de poser la question en «par qui »si l'agent de l'action en question est le seul envisageable ; dans ce cas, on aurait plutôt une question en qui, comme en (8) : l'agent y sera aussi une information nouvelle mais qui est plus neutre.

Ainsi, il devient légitime de postuler que le complément d'agent par $N_{l}$, dans les $P P$, est susceptible d'une certaine lecture "polyphonique », au même titre que la négation, par exemple, qui évoque généralement la contrepartie affirmative, le point de vue assertant le contraire, puisque $\operatorname{par} N_{l}$ ne se contente 
pas d'annoncer l'agent de l'action, comme le ferait le sujet d'une phrase active, mais il vient rétablir, du point de vue du locuteur, une information qui semble échapper à l'interlocuteur, soit parce que l'agent en question n'est pas évident (cela sort de ce qui est canonique), soit il est opposé implicitement ou explicitement à d'autres agents possibles pouvant parfois être de meilleurs candidats pour remplir cette fonction.

\section{ET SI LE COMPLÉMENT D'AGENT N'ÉTAIT PAS SUPPRIMABLE DANS LES PP ACHEVÉES ?}

En passant d'une $P A$ à une $P P$, il est souvent constaté que la permutation qui s'opère au niveau des arguments entraîne un inversement prédicatif, mettant en avant le $N_{l}$ de la $P A$ et faisant de son $N_{0}$ un complément dont on peut se passer la plupart du temps dans le nouvel énoncé passif. Ce constat semble faire l'unanimité dans la littérature sur le passif : l'AP contrairement au $S A$, est la plupart du temps supprimable (Gaatone, 1998 : 182). C'est surtout ce qui arrive au nouveau thème promu au rang de sujet grammatical qui compte ( $c f$. Wilmet, 1998 : 269) ; l'AP serait quelque part une information secondaire dont on peut faire l'économie (Guentchéva \& Desclés, op.cit.) et Brahim (1998 : 291) parle d'une certaine « occultation »; l'agent, selon l'auteur, « tombe dans l'oubli ».

Mais, à bien y réfléchir, quand un complément d'agent est précisé dans une $P P$ alors même qu'il est facultatif, cela éveille des doutes quant à son caractère facultatif et à la possibilité de le supprimer dans une vraie situation de communication, et l'on se demande, en revanche, si l'AP dans les $P P$ achevées ne constitue pas, tout compte fait, le vrai enjeu du prédicat au passif, ce qui rendrait sa suppression, dans un certain sens, impossible! On ne parle pas ici d'une suppression qui entraînerait nécessairement une phrase agrammaticale du point de vue syntaxique, mais d'une suppression impossible du point de vue pragmatique, étant donné que l'intention du locuteur s'en trouve sensiblement affectée et le propos changerait complètement!

Il est vrai que dans beaucoup de cas le complément d'agent peut être soustrait à la $P P$ sans qu'il y ait une perte de sens considérable. Ainsi, l'AP n'est pas précisé, en principe, quand l'information apportée par le " complément d'agent » est «banale/connue », comme dans (8) ci-dessus, ou aussi dans les différents cas répertoriés par Le Goffic (1970 : 84-85) : cela coïncide avec un agent qui n'est pas au centre de la conversation, comme en (10-12), ou correspondant, en général, au sujet on dans leur version active, comme (12) :

10. Je me suis fait couper les cheveux [par le coiffeur].

11. Le nouvel ambassadeur a été reçu à l'Elysée [par le Président de la République].

12. Je me fais comprendre [par « on », on me comprend] (Le Goffic, op. cit. : 83). 
Cependant dans le cas où l'énonciateur éprouverait le besoin de préciser l'agent dans une $P P, l^{\prime}$ 'AP prend une valeur informative importante et n'est plus, en fait, une information banale ; le locuteur présume que l'interlocuteur ignore cette information et c'est d'ailleurs la raison d'être de l'énoncé passif avec un complément d'agent exprimé. Ainsi, si l'agent est précisé dans une $P P$, c'est qu'il est supposé inconnu, ou n'allant pas de soi et devient ainsi indispensable. La précision de l'agent peut servir à rectifier une erreur ou devancer un certain raisonnement conventionnel, qui est, par ailleurs, légitime en apparence ; par ailleurs, cela peut venir tout simplement pointer du doigt l'agent responsable, en l'extrayant d'un ensemble d'agents possibles. De ce point de vue, la réalisation de l'AP en surface vient « singulariser » cet agent ou contrer une inférence établie par défaut et prend alors ce que nous appelons une « valeur contrastive » et dont on ne peut se passer si l'on veut rendre compte fidèlement des intentions de l'énonciateur. Considérons les exemples (13 et 13a). Le complément d'agent par des cambrioleurs pour les procès être cambriolé ou se faire cambrioler paraît de fait redondant ; il relève d'une information que l'on peut qualifier de «banale» (selon les stéréotypes, on est toujours cambriolé par des cambrioleurs inconnus), ce qui équivaut dans la $P A$ au «pronom indéfini » (on) ou au nom cambrioleurs, spécifié par un «déterminant indéfini pluriel »(des); de fait, le préciser rendrait l'énoncé quelque peu bizarre et superflu, si la situation ne l'impose pas autrement, comme dans le cas où le $\mathrm{N}$ est modifié (13b) :

13. L'appartement de Léa a été cambriolé $(\emptyset+$ ?par des cambrioleurs)

13a. Léa s'est fait cambrioler $(\varnothing+$ ?par des cambrioleurs)

13b. Léa s'est fait cambrioler par des cambrioleurs professionnels/amateurs/ parisiens

Cependant, si ce cambriolage a été fait par le petit ami de Léa ou son voisin ou quelqu'un de sa famille, par exemple, en (13c), ce qui s'oppose à ce à quoi l'on peut s'attendre par défaut selon les savoirs partagés et le «bon sens », le fait de préciser l'agent en question - si le vœu du locuteur est tel - devient alors très naturel du point de vue discursif :

13c. Léa s'est fait cambrioler par (son petit ami + sa sœur + son voisin).

L'AP, ici, constitue un apport prédicatif non banal, «n'allant pas de soi », puisqu'il apporte une information importante qui «contraste » avec ce à quoi l'on s'attend normalement et l'énoncé en question admettrait alors des ajouts et des enchaînements du type : Tu te rends compte ?/ Qui l'aurait cru ?!/ Ça alors! Ça par exemple! Où va-t-on ?! Et puis quoi encore ?!. Par conséquent, si l'énoncé de base est (13b), il devient quelque part impossible de supprimer l'AP (son petit ami) si l'on veut être fidèle à la fonction « informative » de l'énoncé et aux intentions de l'énonciateur ; cet énoncé renferme, en fait, une double prédication : (1) « le fait que Léa s'est fait cambrioler » et (2) « Le fait 
que, contrairement à toute attente, c'est son petit ami qui l'a cambriolée (Y) et non un cambrioleur quelconque $(\mathbf{X})$ », là où l'énoncé sans agent (Léa s'est fait cambrioler) ne fait que rapporter « ce qui est arrivé à Léa » tout en laissant entendre qu'il s'agit d'un cas ordinaire (« des cambrioleurs inconnus/non identifiés » équivalents au sujet indéfini on ou des cambrioleurs dans la $P A$ ).

Dans ce qui suit, nous allons nous intéresser de plus près à cette valeur de « contraste » qui nous paraît traverser a priori tous les énoncés de notre corpus où l'on a un «agent passif » explicite en par. Nous essayerons alors de voir à chaque fois comment cette valeur contrastive se manifeste dans le discours.

\section{FIGURES DU «CONTRASTE » DANS LE CORPUS ORAL DE PP}

L'étude des manifestations de la notion de « contraste » à partir des bases des données orales retenues dans cette étude a permis de distinguer, globalement, trois grands cas de figure, avec beaucoup de chevauchements, vu l'effet de «singularisation » qu'ils concourent tous à produire, et qui viennent corroborer le fait que la mention du complément d'agent dans un énoncé passif « achevé » n'est pas facultative du point de vue de l'usage, étant donné qu'elle constitue, tout compte fait, l'information centrale ( $c f$. supra), selon laquelle on établit un contraste entre un agent désigné et approuvé par l'énonciateur (Y) et un ou plusieurs autres que ce dernier écarte $(\mathbf{X})$. Le contraste dans ces énoncés est avant tout exprimé par le segment par $N_{l}$, comme nous l'avons montré plus haut, mais très souvent on trouve d'autres indices textuels qui viennent conforter cette idée de contraste et relèvent globalement de trois types :

(1) Par $N_{l}$ renvoie à un « agent » ignoré, méconnu ou inconnu de l'interlocuteur ou supposé tel du point de vue de l'énonciateur ; on le retrouve dans la distribution des tâches ou dans la désignation " exclusive » d'un écrivain, d'un créateur, d'un architecte, d'un inventeur, etc., dans des messages à fonction informative ou didactique. Ce qui différencie l'AP du $S A$ dans ce type d'énoncés est le fait que l'indication de l'agent en question ( $\operatorname{par} N_{l}$ ) s'opère d'une manière négative et différentielle ; $N_{l}$ fait partie d'un ensemble d'agents possibles exprimés explicitement ou qui sont décelables à partir de l'énoncé.

(2) $\operatorname{Par} N_{l}$ relève d'une réaction de la part de l'énonciateur vis-à-vis d'un constat, d'une attitude, ou d'un énoncé entendu dans un cadre dialogique et interactionnel. L'énoncé passif achevé vient fournir une réponse demandée par le co-énonciateur ou alors vient reprendre ce dernier afin de rétablir les faits mépris ou erronés.

(3) Le troisième cas de figure rejoint le précédent, étant donné que l'énonciateur y vise également à rectifier une idée ou des faits qu'il considère comme erronés, mais, en l'occurrence, l'erreur n'a pas été 
effectivement exprimée par le co-énonciateur, mais relève d'une erreur " latente », d'une induction en sursis, qui s'appuie sur une certaine « logique commune », sur les stéréotypes, le $O N$-locuteur. Par $N_{l}$ vient alors rectifier cette erreur par anticipation, ce que corroborent les multiples procédés polyphoniques utilisés.

Dans tous ces cas de figure, l'agent est une information nouvelle ou supposée l'être du point de vue du locuteur et le complément d'agent par $N_{l}$ révèle contrastivement l'existence d'autres agents possibles. La prise en compte de la dimension dialogique et transphrastique permettra de faire la lumière sur les éléments du cotexte qui mettent en évidence cet aspect « contrastif » dans la démarche adoptée ici. Comme ces différents aspects peuvent se croiser et cohabiter dans un même énoncé, ils seront abordés et classés en fonction de leur saillance. Notre étude de corpus a concerné plus de cent-vingt énoncés passifs, mais faute de place, l'analyse ne concernera que les énoncés ci-après qui nous paraissent représentatifs des trois cas de figure décrits ci-dessus.

\subsection{LE CONTRASTE COMME EXPRESSION D'UN AGENT EXCLUSIF, SORTANT DU LOT}

Soit le corpus allant de (14) à (17). Dans ces quatre énoncés, la mention de l'agent correspond à la révélation d'une information censée nouvelle relativement à un agent censé ignoré ou inconnu ; de fait, le locuteur suppose que cette information est nécessaire et juge bon de la communiquer. Dans ce premier cas de figure, le contraste dont il est question concerne l'opposition explicite entre différents agents possibles. La précision de l'agent vient alors singulariser un élément $\mathbf{Y}$ contrastant avec un autre agent possible $\mathbf{X}$ :

14. BA725: [...] un mouvement comme ça ne peut pas se déclencher avec cette rapidité s'il n'y a pas un mécontentement général n'est-ce pas? mais alors évidemment après j'ai vu quand même que les les les les à enfin à mon avis les extrêmes les extrêmes menaient la la danse alors là j'ai vu que le la majorité était embarquée dans une affaire que euh qui n'était pas prévue au départ certainement euh par les par les pondérés par les les gens qui voulaient continuer d'étudier dans de meilleures conditions n'est-ce pas?

OU : Oui

15. TS165: moi je crois que c'est à mon point de vue c'est ce sont toujours des extrémistes soit de droite soit de gauche

JK: oui oui oui

TS165: qui sèment le désordre et puis les autres ils sont entraînés ils sont entraînés par ces par ces gens-là quoi

16. JSM: hm hm vous ne voyez rien contre enfin une femme mariée avec des enfants qui travaille c'est?

HU339: ah à partir d'un certain âge il faut quand même que la femme 
s'occupe de ses enfants là c'est une raison d'éducation si c'est élevé par une nourrice c'est sûrement pas euh une éducation normale

JSM: $\mathrm{hm}$ hm et mais quand même euh

HU339: c'est-à-dire entre quatre et dix ans euh au point de vue âge de l'enfant

JSM: $\mathrm{hm} \mathrm{hm}$

HU339: à mon avis faut que la femme reste avec eux

JSM: reste avec les enfants et puis plus tard

HU339: \#1 enfin elle recommence à travailler après \#

JSM: \#2 oui reprendre \# le travail [tx]

17. IZ248: \#2 oui oui \# oui en principe alors il y a des écoles qui sont faites exclusivement par des religieuses ou certains par des prêtres mais alors maintenant ils ont beaucoup d'institutrices euh civiles qui sont dedans

Notons que les deux premiers échanges (14) et (15) extraits des ESLO tournent autour des " événements 68 » (on demande aux interviewés de dire ce qu'ils en pensent, de quelle manière ils ont vécu ces événements qui étaient encore d'actualité au moment de l'interview). Tout d'abord, l'énoncé (14) rapporte le point de vue de l'interviewé quant à la responsabilité vis-à-vis du déclenchement des événements 68 en France et plus précisément à Orléans, repris sous le $N_{l}$ (une affaire) dans [...] une affaire que euh qui n'était pas prévue par les pondérés... ; l'énonciateur livre ainsi ses pensées, ses convictions personnelles, en écartant l'un des agents possibles $\mathbf{Y}$, pourtant pris pour le vrai responsable par l'opinion publique, à savoir « les étudiants » (les pondérés par les les gens qui voulaient continuer d'étudier dans de meilleures conditions), contrastant avec les « extrêmes » (les extrêmes les extrêmes menaient la la danse) et vient rétablir ainsi une vérité paraissant, de son point de vue, non évidente, en niant l'implication des étudiants ( $c f$. la négation : n'était pas prévue par les pondérés...), et en incriminant un agent non évident, les extrêmes, « ceux qui tirent les ficelles/qui manipulent les étudiants innocents de loin » (...la majorité était embarquée dans une affaire que euh qui n'était pas prévue...). D'ailleurs, l'antithèse «pondérés » versus « extrêmes » est le meilleur porte-parole de cette idée de contraste, sans parler du caractère polyphonique de la négation utilisée, qui est de nature à rappeler que contrairement à ce que laissent croire les apparences, les étudiants ne cherchaient pas à en arriver là.

Ainsi, l'agent passif (par les pondérés par les les gens qui voulaient continuer d'étudier dans de meilleures conditions) présent dans l'énoncé (14), relève d'un cas de ce que nous appelons une « singularisation » de l'un des agents possibles vis-à-vis du procès « déclenchement du mouvement 68 » : on pointe du doigt un membre d'un ensemble susceptible d'occuper ce rôle et qui est incriminé à tort, comme le montre la forme négative. Cette singularisation se 
trouve renforcée par une disfluence en (14) qui tient lieu, en fait, d'une relative apposée, qui n'annule pas le $S P$ précédent (par les pondérés...), mais le précise davantage pour le faire sortir du banc des accusés et le disculper définitivement : ils ont d'autres préoccupations ( «les études »), avec la reformulation par les les gens qui voulaient continuer d'étudier dans de meilleures conditions. Il en va de même de l'exemple (15), qui s'inscrit dans le même sujet de conversation (les événements 68) et dans une même attitude que (14), vis-à-vis de l'implication apparente des étudiants : on oppose, d'un côté, les étudiants (les autres), qui sont présentés comme victimes d'une manipulation, comme ceux qui suivent contre leur volonté (les autres sont entraînés), de l'autre, les extrémistes (par ces gens-là quoi) qui est une reprise anaphorique du segment (des extrémistes soit de droite soit de gauche... qui sèment le désordre).

Les exemples (16) et (17) mettent en scène le même type de « singularisation » en décrivant des situations différentes s'inscrivant dans la mentalité des années $68:$ le locuteur oppose explicitement deux méthodes d'éducation des enfants en bas âge en (16) et deux types d'écoles en (17). Dans le premier énoncé, on distingue une première méthode que le locuteur juge normale et naturelle (il faut quand même que la femme s'occupe de ses enfants...) et une deuxième qu'il juge inappropriée (par une nourrice) et, dans le dernier, l'opposition concerne " l'école tenue par des religieuses ou des prêtres » d'un côté, et « celles qui sont tenues par des institutrices » de l'autre. Le contraste, dans les exemples (14-17), renvoie ainsi à des agents explicites qui s'interdéfinissent différentiellement les uns les autres. Ce contraste se trouve souligné par tout une batterie d'indices textuels : on a d'une part, des procédés comme l'antithèse au niveau des agents (extrémistes/extrêmes vs étudiants); (mère vs nourrice) ; (religieuses/prêtres) vs (institutrices), ou aussi la négation en (14); d'autres part, l'usage de mais en (17) et du marqueur et puis en (15) qui ont une valeur « adversative », « disjonctive » et " contrastive ». Outre ces différents marqueurs discursifs, il y a aussi tous les procédés prosodiques, caractérisant le discours oral, qui concourent à leur tour à la réalisation de cet effet contrastif. Ainsi, dans l'exemple (17), le contraste explicite entre agents possibles est renforcé par une intonation montante au niveau du segment (faites exclusivement par des religieuses ou certains par des prêtres) par opposition aux « écoles avec des institutrices » où l'on a plutôt une intonation descendante (ça ne fait que rappeler un agent plus connu, plus ordinaire). On distingue également ce qu'on appelle « accent contrastif » dans l'exemple (14) couvrant la négation de l'agent présumé. Par ailleurs, dans tous ces exemples, la tension est maintenue jusqu'à la fin du $S P$ servant de complément d'agent, ce qui prouve que le locuteur insiste davantage sur cette partie de l'énoncé qui renferme une information qui ne va pas de soi et qui mérite d'être écoutée attentivement par le co-énonciateur. 


\subsection{LE CONTRASTE DANS LES PP ACHEVÉES MIS À NU PAR LES MARQUEURS DIALOGIQUES}

Le deuxième cas de figure où l'on peut relever l'idée de contraste entre les agents possibles vient des interactions dialogiques entre interviewé et interviewer. En effet, chacun de ces deux acteurs se positionne par rapport à ce que l'autre dit, présuppose ou laisse sous-entendre dans leurs échanges. En d'autres termes, l'énonciateur réagit à ce qu'il entend : $\operatorname{par} N_{l}$ spécifie «l'agent » dans le cadre d'une réponse à une question sur les agents éventuels ou face à une méprise entendue/constatée et l'énoncé passif vient ainsi rétablir les faits manquants, ou rectifier l'erreur perçue, etc. Considérons les exemples (18-20) :

18. JSM: et euh on est-ce que vous sa-est-ce que vous savez si vos parents lui ont acheté le dictionnaire ou si c'était un cadeau ou TN153: alors là non c'est été ça a été donné par l'école JSM: donné par l'école

TN153: oui ça été donné par l'école oui c'est des dictionnaires qu'ils donnent à l'école

19. BV: on a beaucoup parlé des événements de mai juin euh les Anglais étaient évidemment pas là en France à ce moment-là est-ce que vous pourriez décrire enfin pour les Anglais qui vont écouter les bandes ce qui s'est passé en France à ce moment-là?

MD461: difficile ce que vous me demandez là

BV: hein ? est-ce que vous pourriez décrire enfin ce qui s'est passé en France quoi? parce que vous savez que les bandes vont partir après en Angleterre qu'elles seront écoutées par les étudiants euh

20. FC716: celui qui sait pas écrire à quatorze ans ben il saura jamais écrire il fera toujours les mêmes fautes à vingt-cinq ans

DP: oui oui vous trouvez pas ça euh \#1 très simple euh? \#

FC716: \#2 ah si si \# si ça euh vraiment je suis pas je suis pas pour ça quand même parce que j'estime quelqu'un qu'est euh qui va être appelé à occuper de grands emplois bah ma foi

DP: oui

FC716: $i$ - il est intraitable qu'ils se fassent corriger des fautes par des subordonnés

L'exemple (18) met en scène la situation la plus banale du contraste qui s'inscrit dans le dialogisme. Il s'agit tout simplement d'une réponse à une question, doublée d'une dénégation de ce qui est suggéré explicitement par l'interviewer comme agents possibles : l'interviewé écarte les propositions avancées par hypothèse par le co-énonciateur ( « ce serait les parents qui auraient acheté le dictionnaire » et « ce serait un cadeau ») et rétablit le vrai agent (l'école) : alors là non c'est été ça a été donné par l'école et le rapport 
contrastif vient ipso facto de cette relation entre les agents devinés ( $\mathbf{X}$ donnés explicitement) et celui qui est présenté dans l'énoncé passif comme le vrai agent (Y) et qui ne fait pas justement partie des prévisions de son interlocuteur.

Les interactions dialogiques, en (19), révèlent un autre type de situation : l'interviewer réagit face à une gêne ressentie par l'interviewé à l'égard du caractère qu'il estime indiscret et compromettant de la question posée (difficile ce que vous me demandez là) ; cela porte sur ses opinions politiques, plus précisément, la question invitait l'interviewé à s'exprimer sur un sujet d'actualité, les événements 68, alors que la situation en France à l'époque était encore loin d'être stable, à plus forte raison, la conversation allaient être enregistrée. L'interviewer essaye de réconforter son interlocuteur en lui assurant que les bandes magnétiques allaient être écoutées «par des Anglais et non par des Français et qu'elles auraient un usage purement pédagogique » (parce que vous savez que les bandes vont partir après en Angleterre qu'elles seront écoutées par les étudiants euh). Ainsi, le contraste est établi entre ce que laisse entendre le locuteur avec son attitude embarrassée ( $c f$. ses pensées peuvent ne pas être partagées par les autres Français qui pourraient écouter ces bandes, ou par le Gouvernement français, lui-même, ce qui lui serait préjudiciable) et la vraie finalité des enregistrements (servir de supports pédagogiques pour des anglophones qui apprennent le français comme langue étrangère). Il en va de même pour l'exemple (20). Cette fois-ci, c'est une réaction face à une attitude de « déni » devant une assertion qui s'avère pourtant vraie, à laquelle adhère l'énonciateur. En effet, l'interviewé prétend que celui qui fait des fautes, jeune, est condamné à en faire même quand il devient adulte (celui qui sait pas écrire à quatorze ans ben il saura jamais écrire il fera toujours les mêmes fautes à vingt-cinq ans); l'interviewer semble trouver cette affirmation quelque peu abusive (oui oui vous trouvez pas ça euh \#1 très simple euh?\#), ce que pouvait d'ailleurs pressentir l'interviewé lui-même qui semble s'y attendre en complétant lui-même la phrase amorcée sous forme d'une question (\#1 très simple euh?\# $\rightarrow$ usage des dièses (\#) pour marquer les chevauchements des tours de parole) et c'est ainsi que la réplique de ce dernier cherche à prouver qu'il a raison de dire ce qu'il dit à travers l'emploi dialogique de si qui sert, ici, à confirmer une négation (ah si si si), ainsi qu'à travers l'antithèse établie entre des personnes occupant de hauts rangs (quelqu'un qu'est euh qui va être appelé à occuper de grands emplois) et qui se font aider par des personnes qui sont censées être moins bons qu'eux (des subordonnées) : $i$ - il est intraitable qu'ils se fassent corriger des fautes par des subordonnés. Ce sont ainsi les interactions énonciatives qui font avancer le discours, dans ces exemples, et qui suscitent le recours aux phrases passives achevées où l'agent relève d'une donnée importante contrastant avec le crédo du co-énonciateur. 


\subsection{LE CONTRASTE DANS LES PP ACHEVÉES À TRAVERS LES PROCÉDÉS POLYPHONIQUES}

En continuité avec les exemples précédents, les énoncés allant de (21), (22) et (23) véhiculent un contraste, qui concerne cette fois-ci des agents implicites, non mentionnés en surface, mais dont l'affirmation est indéniable si l'on se fie aux marqueurs polyphoniques présents dans l'entour discursif considéré : le locuteur désigne l'agent pour ôter un doute, rectifier une erreur, etc. par anticipation ; il se fonde sur une certaine logique imposée par l'habitude et « la force des choses », un certain « bon sens », relevant du « non-dit » et correspondant globalement à la notion de « $O N$-locuteur(s) » :

21. GX120MAR: nous suivons nous suivons comme tous les français les discussions qu'y a entre les états-majors des différents partis politiques qui constituent la gauche française et on voit que ces hommes ont beaucoup de mal à se mettre d'accord pour arriver à fonder un parti commun de la gauche française

PB: hm

GX120MAR: on le constate et on le déplore en même temps

PB: hm hm hm hm

GX120MAR: c'est tout et puis ce que je vous dis là c'est partagé par beaucoup d'électeurs français

22. HN169: or il y a une impossibilité absolue entre ces deux impératifs ou vous faites beaucoup de réalisations et vous écrasez la population d'impôts

JSM: $\mathrm{hm} \mathrm{hm}$

HN169: ou vous ne leur faites pas payer d'impôts mais alors euh y a aucune réalisation je crois que dans ces deux dans cette alternative il y a une demi-mesure qui pourrait être quand même une solution euh Orléans s'est trouvé en pleine crise de croissance je vous l'ai dit à cause de La Source je crois que le maire d'Orléans a été en partie trompé par les pouvoirs publics quand il a en somme lancé l'opération La Source il comptait sur une participation beaucoup plus importante du département et de l'État

JSM: ah oui

23. JO304: quand on quand on voit certains certains textes euh qui ont été euh certaines paroles qui ont été dites par des ministres

086LOC1?086LOC2: [pron=pi]

JO304: et bien j'avoue vraiment que on peut un peu désespérer de l'avenir euh intellectuel des des enfants qui viendront

Dans l'échange en (21), après avoir parlé de son indignation de l'éclatement de la gauche française, le locuteur GX120MAR devance une éventuelle 
méprise de ses propos, et tente alors de mettre les points sur les $i$, au sujet de ce qui pourrait passer pour une attaque gratuite et injustifiée de sa part contre la gauche française ou pour un aveu concernant ses positions politiques, quand il a évoqué "l'incapacité des partis de gauche de s'unir », et lui assure que ce qu'il dit est loin d'être une fantaisie, d'une excentricité et que cela relevait plutôt d'une conviction que partagent beaucoup de citoyens (c'est tout et puis ce que je vous dis là c'est partagé par beaucoup d'électeurs français); c'est ce que pourrait confirmer l'ajout en (21a) qui est de nature à expliciter cette « anticipation » :

21a. N' imaginez pas que j'invente ou que ce soit une chose marginale, ce que je vous dis là c'est partagé par beaucoup d'électeurs français...

Le locuteur écarte une inférence possible que pourraient laisser entendre ses propos et rétablit celle qui présente, de son point de vue, la vérité à retenir. Dans (22), la valeur contrastive vient de l'opposition entre ce qui est connu et communément attendu, d'un côté, et ce qui s'est réellement produit de l'autre : selon les savoirs partagés, le maire n'est autre que l'un des représentants locaux des pouvoirs publics, au niveau communal ; or, la situation renvoie dans cet énoncé à une certaine « trahison»: « les pouvoirs publics auraient trompé l'un des leurs, le maire »; personne ne pouvait s'y attendre (je crois que le maire d'Orléans a été en partie trompé par les pouvoirs publics quand il a en somme lancé l'opération La Source il comptait sur une participation beaucoup plus importante du département et de l'État). On pourrait insérer une apposition visant à plaindre le Maire (le pauvre) ou enchaîner avec un ajout de phrase exprimant la surprise ou la déception Qui l'aurait cru ?!, comme en (22a) :

22a. [...] je crois que le maire d'Orléans, le pauvre, a été en partie trompé par les pouvoirs publics! (Qui l'aurait cru?!).

Il en va de même pour (23), où il est question de la manière dont les gens parlent français ; le contraste y concerne la qualité du discours oral ou écrit de certains ministres (Y) (quand on voit certains certains textes euh qui ont été euh certaines paroles qui ont été dites par des ministres) et que l'interviewé considère comme indigne du rang social des ministres; ces derniers se trouvent opposés à des agents non-dits renvoyant à toute autre personne n'étant pas ministre ou n'ayant pas la formation d'un ministre $(\mathbf{X})$ et dont les propos ne seraient pas déplorables ni choquants pour autant en produisant de tels discours. En effet, on ne peut pas excuser à quelqu'un qui occupe le poste de ministre, donc, quelqu'un faisant partie de l'équipe qui pilote le pays, de parler comme le reste de la population : (et bien j'avoue vraiment que on peut un peu désespérer de l'avenir euh intellectuel des des enfants qui viendront). Comme pour les exemples précédents, les enchaînements possibles sont de nature à mettre en évidence l'attitude du locuteur : Vous vous rendez-compte ?! Vous imaginez?!. 
Notons que dans tous les exemples du corpus oral, le complément d'agent n'est pas une donnée facultative comme on le fait souvent remarquer dans la littérature sur la question. La présence de l'AP constitue, somme toute, l'enjeu principal de l'énonciation sur le plan prédicatif. Les phénomènes prosodiques sont de nature à confirmer ce fait, puisque l'énonciateur met plus l'accent sur le segment $\operatorname{par} N_{l}$, ainsi que les segments qui s'y rattachent sémantiquement ou syntaxiquement, à travers la saillance et la tension au niveau des schémas mélodiques. D'ailleurs le relâchement au niveau de la prononciation de ces segments ne serait sans doute pas naturel.

\section{CONCLUSION}

Le recours à un corpus de données orales mettant en scène des énonciateurs s'exprimant d'une manière non préméditée, doublé d'une approche de type dialogique et macrosyntaxique, dépassant le cadre de la phrase simple et simplifiée, aura permis, dans cette étude, de mieux saisir les nuances sémantiques et pragmatiques sous-jacentes à l'emploi d'un énoncé passif achevé plutôt que d'un énoncé actif, et plus avant celles qui sous-tendent l'utilisation d'un complément d'agent en par, par opposition au sujet actif correspondant du point de vue transformationnel. Notre étude aura montré que l'utilisation d'un $A P$ permet d'instaurer une « singularisation» de l'agent qui se traduit à travers l'expression d'un certain « contraste » qui s'opère entre les agents possibles pour un procès donné. Plus précisément, il se manifeste entre ce qui est écarté comme information concernant l'« agent » - quoique pouvant paraitre vraisemblable et ce qui est validé et rapporté par l'énonciateur quoique paraissant douteux et improbable. Cette idée de contraste génère différents types d'effets pouvant aller de la simple précision d'un élément qui se distingue du lot à la désignation d'un élément « surprenant», « inattendu » et « non-canonique » contrastant avec l'élément qui se présente à l'esprit par défaut. Cette valeur de contraste, qui traverse selon nous tous les énoncés passifs achevés, a pu être décelée, dans cette étude, grâce aux interactions énonciatives observables à l'oral dans notre corpus, à travers différents marqueurs discursifs polyphoniques et dialogiques. Il en ressort que bien que certaines $P P$ et $P A$ soient liées du point de vue sémantique et syntaxique, elles appartiennent, en revanche, à deux univers discursifs différents, compte tenu du type d'information que véhicule chacune des ces deux formes sur le plan pragmatique. Un tel résultat permet ainsi de mettre en cause l'hypothèse de l'identité sémantique $A P-S A$, et audelà, $P P-P A$. Il ne serait plus judicieux, par conséquent, d'établir des rapports de correspondance actif-passif sans tenir compte des enjeux discursifs que ces changements de forme entraînent. 


\section{BIBLIOGRAPHIE}

Anscombre, J.-C. (2005), « Le $O N$-locuteur : une unité aux multiples visages », in Bres, J. et al., Dialogisme et polyphonie. Approches linguistiques, De Boeck, Bruxelles : 75-94.

Balthasar, L. et Bert, M. (2005), « La plateforme « Corpus de langues parlées en interaction » (CLAPI) Historique, état des lieux, perspectives », Corpus oraux et diversité des approches, Lidil 31 : 13-33.

Berrendonner, A. (1990), « Pour une macro-syntaxe», Travaux de linguistique 21 : 25-36.

Blanche-Benveniste C. (1988), « La notion de contexte dans l'analyse syntaxique des productions orales : exemples des verbes actifs et passifs », Recherches sur le français parlé $8: 39-57$.

Blanche-Benveniste C. (2000), « Analyse de deux types de passifs dans les productions de français parlé», Études Romanes, $n^{\circ}$ spécial, Actes du colloque international Institut d'Études Romanes, Université de Copenhague (5-7 mars 1998), Lene Schosler (éd.), Le passif 45 : 303-319.

Bres, J. (2005), « savoir de quoi on parle : dialogue, dialogique ; dialogisme, polyphonie... », in Bres, J. et al. Dialogisme et polyphonie. Approches linguistiques, De Boeck, Bruxelles : 47-62.

Carlier, A. (2002), «Les proprié tés aspectuelles du passif », Cahiers Chronos 10: 41-63.

Eshkol-Taravella, I. et al. (2011), « Un grand corpus oral « disponible » : le corpus d'Orléans 1 1968-2012», in TAL. Volume 53 - $n^{\circ} 2$ : 17 à 46.

François, J. (1989), Changement, causation, action: Trois catégories sémantiques fondamentales du lexique verbal français et allemand. Librairie Droz.

Gaatone, D. (1993), «Les locutions verbales et les deux passifs du français ». Langages 27, Larousse : 37-52.

Grice, H. P. (1975), Logic and conversation (trad. 1979), Éditions du Seuil.

Guentchéva, Z., \& Desclés, J.-P. (1993), « Le passif dans le système des voix du français », Langages27, Larousse, 73-102.

Hamma, B. (2005), L'invariant sémantique de la préposition par à travers les distributions syntaxiques et lexicales, Thèse de doctorat, sous la direction de D. Leeman, Paris 10 Nanterre.

Hamma, B. (2006), «État des lieux sur la sémantique de la préposition par », Modèles linguistiques 54, -2, La préposition en français II. Éditions des Dauphins, Toulon : 81-95.

Hamma, B. (2007), « La notion de « contraste » dans les locutions de type par $N »$. Modèles Linguistiques 55 : 1, Éditions des Dauphins. Toulon : 77-92.

Jackendoff, R. (1997), The Architecture of the Language Faculty, MIT Press. 
Jackendoff, R. (2005), « Alternative minimalist visions of language », Chicago Linguistics Society, 41 : 189-226.

Le Goffic, P. L. (1970), « Linguistique et enseignement des langues : à propos du passif en français ». Langue française 8, Larousse : 78-89.

Lebellec, C. \& Hamma, B. (2011), « Le passif en français : de nouveaux éléments de

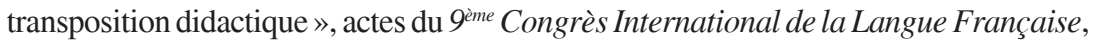
Madrid.

Lebellec, C. \& Hamma B. (2014), « Le traitement du passif dans l'enseignement du français langue étrangère », in Lidil, 50 : 191-211.

Leclère, C. (1993), « Classes de constructions directes sans passif », Langages27, Larousse : 7-31.

Leduc-Adine, J.-P. (1977), « Actif et passif des manuels », Langue française, 33, Larousse : 90-101.

Martin, F. (2002), « La préposition de du “ complément d'agent " des verbes psychologiques causatifs : un génitif ». In Kupferman, L. (éd.), La Préposition dans tous ses états, 4, Scolia $15: 57-70$.

Mathieu, Y. (1993), «Quelques passifs avec agent obligatoire», Langages 27, Larousse : 35-36.

Muller, C. (1977), « Analyses linguistiques des relations de champ entre quantificateurs et négation », Langages 11, Larousse : 60-83.

Strunk Sachs, J. (1967), « Recopition memory for syntactic and semantic aspects of connected discourse », in Perception \& Psychophysics Volume 2, Issue 9 : 437-442. 